\title{
Chapter 5 \\ Discussion and conclusions of the results obtained during the standardization of a real time PCR for Escherichia coli quantification and Salmonella spp
}

\author{
Jairo Ceballos Sandoval \\ Universidad Simon Bolivar, Colombia \\ jceballos1@unisimonbolívar.edu.co \\ Maria Badillo Viloria \\ Universidad Simon Bolivar, Colombia \\ Corporación Universitaria Rafael Nuñez, Colombia. \\ mbadillo3@unisimonbolívar.edu.co
}

The development of the discussion and conclusions will be based on the present research results, seeking to contrast them with the public policies on issues related to water quality and its relation with the National Program of Science and Technology of the Sea and Hydrobiological Resources, (Resolution No. 370 of 2015) that prioritize the CTeI of water in Colombia. The findings derived from the development of the project and a review of the evolution of the National Policy on Science and Technology show a strong incidence on the main landmarks that have been associated with the integral management of water, such as diagnostic methodologies for its quality control.

The entry into force of the General System of Regalias and the respective creation of the Science, Technology and Innovation Fund has opened up an opportunity for science, technology and innovation in the country (90). 
New resources will positively impact the National Program of Science, Technology and Innovation of the Sea and the Hydrobiological Resources and therefore in the application of Water National Policies and those related to its oceans and coasts (91).

At present, only projects associated with aquaculture and ports have been strengthened (92). It is necessary to present projects related to the basic sciences and their direct relation with water, e.g provide data about its quality through molecular tools such as PCR, whose results help making decisions quickly and efficiently, generating interdisciplinary knowledge that contributes to a better Colombian water resources administration.

The analysis of the microbiological indicators of water makes it evident that Colombia has an acceptable water quality, but with traditional indicators of low reliability and poor facilities in public institutions, which allow perform high quality tests and therefore international comparisons. The process of researching molecular tools in Colombia in relation to water quality has not yet been consolidated, many of the drawbacks identified in past diagnoses still persist due to the low levels of innovation of public and private institutions.

The weak institutionality of the system, the lack of focus of the policy in strategic areas, the low social appropriation of knowledge and regional disparities in scientific and technological capacities, which together generates a low capacity to generate and apply basic knowledge in most regions of the country. However, cannot ignore the progress that has been made in relation to the generation of knowledge applicable to science and its relationship with water management, reinforced on the one hand by the increasing presence of highly trained personnel level.

When implementing molecular methods to the diagnosis of water in the laboratories of microbiological can be taken into account the results 
Discussion and conclusions of the results obtained during the standardization of a real time PCR for Escherichia coli quantification and Salmonella spp

obtained in the process of standardization of a PCR technique performed in this research, from which it was evident that the filtration method is the most suitable for the bacterial concentration, because it allows to recover in a greater percentage the amount of microorganisms inoculated in a water sample, comparable with other technologies (93). With respect to the elution, it was established that a mechanical trituration of the filter submerged in Glycine/Peptone buffer obtained a better results of DNA extraction process, after a vortexing between 8 min-10 min that allows the separation and direct extraction of the DNA comparable to the results obtained with tissier and collaborators (94). The incubation time was discarded due to cell growth and therefore it is not possible to know the exact amount of bacteria present.

Based on the above, finally a method was chosen that combines concentration with filtration, elution with filter grinding with the Glycine/Peptone buffer and vortex for 10 minutes. According to this, amplification was possible in drinking water with low microbial load and in the case of sea water also the amplification was achieved but with a greater amount of CFU without being inhibited by any component of sea water. However, the quantitative results, do not allow to associate the value of $\mathrm{Ct}$ with the amount of inoculated bacteria, not observing a linearity when working with low number of CFU; however, other studies have shown better results in relation to the variance of real-time PCR results (59).

The need for quantitative data by microbiological methods can be solved by the real-time PCR variant, by performing a standard curve. In the preliminary tests performed with this objective, the DNA concentrations obtained do not show a trend according to the utilized dilutions of the bacterial inoculum, especially in the case of Salmonella spp., where some samples were negative. However, when developing the real-time PCR with E. coli DNA, certain linearity was observed except for the last dilutions. As found in similar studies (95), the developed curve give limited quantitative data when working with low concentrations. 
In relation to preliminary results for the standardization of the qualitative type method for the detection of Salmonella spp. through conventional PCR amplification, they demonstrated that it was possible to detect the Salmonella spp. invA gene from DNA extracted from a 24 hours pre-enrichment culture of a sample of water with a low microbial load in both drinking water and sea water; the realization of pre-enrichment step is necessary for the detection of Salmonella spp. by PCR, as demonstrated by Villarreal 2008 (96). Likewise, the obtained data evidenced that greater amounts of DNA were obtained with the traditional extraction method with chloroform than with commercial extraction kit; however, the DNA obtained in the latter was of better quality, this has also been demonstrated by other studies (97).

In relation to the evaluation of the real-time PCR method for the detection of Salmonella spp., a good reproducibility was demonstrated with small standard deviations in both drinking water and sea water using low microbial loads. Real-time amplifications show that there were no noticeable differences between the Cqs obtained by both DNA extraction methodologies. The above demonstrates that it is possible to apply the PCR technique in real time to obtain quantitative and qualitative data; however, in the first case it is limited with low microbial loads, although it is possible to detect bacterial loads of $1 \mathrm{CFU}$ as evidenced by similar studies $(7,96)$.

From these results it is possible to infer that the molecular methods are useful for the identification of pathogenic of rapid, sensitive and specific form; the characteristics of performance of the used methods make it useful to the evaluation of the drinkable water quality and sea water in in all the regions of the country. This work can be the base so that the public health institutions are to the avant-garde in diagnosis methods, which allows improving the alertness of these pathogenic in water sources and 
Discussion and conclusions of the results obtained during the standardization of a real time PCR for Escherichia coli quantification and Salmonella spp

diminishing the risks for the human and animal health, associated with the consumption of contaminated water.

For the Colombian government, the identification of the interdisciplinary research priorities for the integral management of water in the country (98) should be a permanent task, through projects that allow the understanding and diagnosis of the complex processes of state changes, trends and impacts in spaces where the water resource is fundamental. To achieve this goal, regional governments should strengthen both the implementation of molecular tools, qualified personnel and the strengthening of their facilities within the surveillance institutions (Departmental Public Health Laboratories), which create an evident culture of science with participation of all stakeholders, without leaving aside the communities that live and depend directly on the supply of goods and services of the water resource, and achieve greater environmental awareness among citizens.

It is believed that these issues are of a structural nature to support the future of good water governance in Colombia, characterized by its social justice and environmental sustainability. This will be achieved by the design of new innovative networks in scientific research, with a greater interdisciplinary and respectful character. Such processes will require profound transformations of the educational system, whose objective is to train researchers and professionals capable of understanding, listening and dialogue with others socio-cultural groups with a vast knowledge and a diverse traditional practices to generar new strategies that improve the water quality (99).

\footnotetext{
How to quote this chapter:

Ceballos Sandoval J, Badillo Viloria M. Discussion and conclusions of the results obtained during the standardization of a real time PCR for Escherichia coli quantification and Salmonella spp. In Badillo Viloria M, Pérez Lavalle L, editors. Realtime PCR applied to bacterial waterborne pathogens detection and quantification. Barranquilla: Ediciones Universidad Simón Bolívar; 2018. p. 71-75.
} 